\title{
Wireless Sensor Network Using Energy Efficient Ring Routing Protocol with Mobile Sink
}

\author{
Sudharson .S ${ }^{1}$, Jennie Bharathi .R, M .E $\mathbf{E}^{2}$ \\ ${ }^{1}$ PG Scholar, Department of ECE, Sri Krishna College of Engineering and Technology, Kuniamuthur P.O., Coimbatore-641008, Tamil \\ Nadu, India \\ ${ }^{2}$ Assistant Professor, Department of ECE, Sri Krishna College of Engineering and Technology, Kuniamuthur P.O., Coimbatore-641008, \\ Tamil Nadu, India
}

\begin{abstract}
In wireless sensor networks efficiency of energy is considered to be important role due to the limited battery capacity of sensor nodes. In existing system various hierarchical approaches have been experimented, in which each approach suffers from overhead, hotspot and flooding problem. In this paper we propose ring routing approach energy-efficient mobile sink routing protocol is introduced, which aims to minimize this overhead while preserving the advantages of mobile sinks. This ring routing approach is designed to support multiple mobile sink. The technique forms a ring of nodes from the available regular nodes. Ring is formed with the help of certain radius from the centre, nodes closer to the ring which is defined by the radius is formed. The location of the sink node is found through a ring node and is shared between all the ring nodes. Then the source node with the data forwards it to the sink through the anchor nodes.The proposed system achieves higher performance, lifetime and a smaller amount delay while compared with the existing system and this system prevents congestion, traffic between the nodes in case of large wireless sensor network.
\end{abstract}

Keywords: Anchor Node, Flooding, Mobile sinks, Ring Routing, Overhead.

\section{Introduction}

\subsection{Introduction about Wireless Sensor Network}

A Wireless sensor network is a special type transducer with a communications infrastructure that is intended to record and monitor conditions at dissimilar locations. generally monitored parameters are temperature, humidity, wind direction and speed, intensity of illumination, vibration and sound, chemical concentrations, and vital body functions.

\subsection{Overview}

A sensor network which consists of multiple detection stations called the sensor nodes, each of which is small, which is very light in weight and moveable. Each sensor node is equipped with a transducer, microcomputer, power source.

The transducer which generates electrical signals based on sensed physical effect. The microcomputer process and store the sensor output. The power for sensor node is derived from the electrical utility or from a battery. The solutions to the problem is rectified by mobile sink [1],[6],[7],[10].

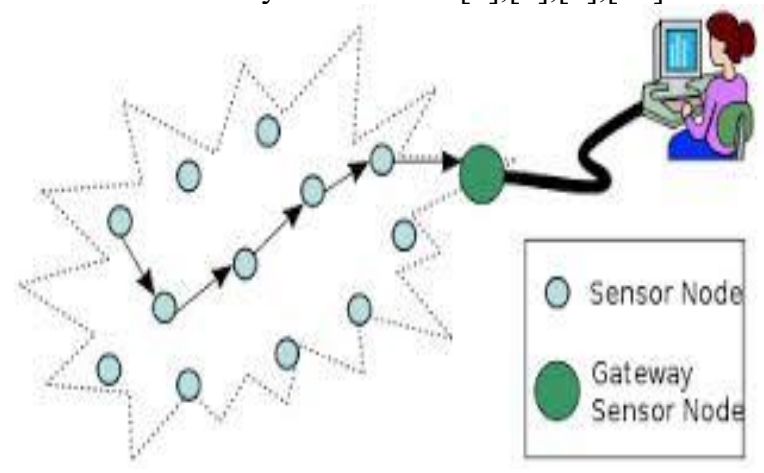

Figure 1.1: Sensor network
The WSN is built of nodes from few to many hundreds or even thousand, where every node is connected to one or several sensors. The size of a grain of dust, although functioning motes of true microscopic dimension have yet to be created. The topology of the WSN can vary from a simple star network to an highly developed multihop wireless mesh network. Size and cost constraint on sensor node result in corresponding constraint on resources such as energy, memory, processing speed and communication bandwidth.

\subsection{WSN Characteristics ad Architecture}

A WSN is a homogenous or heterogeneous system which consists of hundreds or thousands of low cost and lowpower tiny sensors to monitor and gather information from the real time distribution environment. Common functionalities of WSNs' nodes are broadcast and multicast nodes, routing, forwarding and route maintenance. The sensor's components are: sensor unit, processing unit, storage unit, power supply unit and wireless radio transceiver; these units are communicating to each other.

Wireless communications and weak connections, Low reliability and failure capability in sensor nodes, Vibrant topology and self-organization, Hop by hop communications (multi-hop routing), Inter-nodes broadcast-nature communications. 


\section{International Journal of Science and Research (IJSR) \\ ISSN (Online): 2319-7064}

Index Copernicus Value (2013): 6.14 | Impact Factor (2015): 6.391

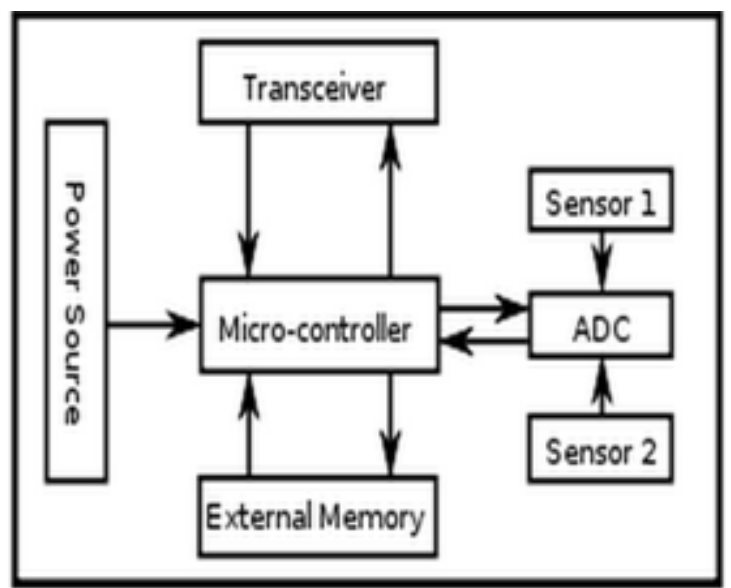

Figure 1.2: Architecture diagram of the sensor node

The main components of a sensor node are a microcontroller, transceiver, memory, power source and more sensors. The controller performs tasks, processes data and controls the functionality components in the sensor node. The functionality of both transmitter and receiver are combined into a single device which is known as a transceiver. The operational state is to transmit, receive, idle, and sleep. Most transceivers which operates in idle mode have a power utilization almost equal to the power consumed in receive mode. External memory is used for storing application that is related or personal data, and program memory used for programming the device. An essential aspect in the development of a wireless sensor node is ensuring that there is always adequate energy available to power the system. The sensor node consumes power for sensing, communicating and data processing. Very high energy is needed for data communication than any other process. Sensors are hardware devices that produce a measurable response to a change in the physical condition like temperature or pressure. Sensors measure physical data of the parameter which has to be monitored. The continual analog signal produced by the sensors is digitized by an analog-to-digital converter converts the data send to the controllers for further processing.

\subsection{Need for Proposed System}

Ring Routing establishes a virtual ring structure that allows the fresh sink position to be easily delivered to the ring and regular nodes to attain the sink position from the ring with minimal overhead whenever needed.

The ring structure can be easily changed. The ring nodes are able to switch roles with regular nodes by efficient mechanism and straightforward mechanism, thus mitigating the hotspot problem. The wireless sensor network is battery operated. So the energy consumption is playing the major role. This work attempts to provide the fast delivery through quick accessibility and it can deal with increasing data rate successfully.

Large sensor network can be managed efficiently as the multiple sink node reduces the energy consumption.Mobile sink can also be used for habitat monitoring, where a robot designated as the mobile sink gathers information from the sensor node which is located in different areas in a large field network [13].

\section{Existing Method}

In a typical wireless sensor network, the batteries of the nodes near the sink drain quicker than other nodes due to the data traffic which is concentrated towards the sink node. To mitigate this problem, mobile sinks are proposed. The existing system introduced different routing protocol approaches with various drawback. The existing approaches are,

\section{1(LBDD)-Line Based Data Dissemination}

An LBDD stand for line based data dissemination defines vertical strips of nodes centered on area of deployment.

The information or data is first sent in to the line, the node which is first present in the line encounter the data. Sink node collects the data from the sensor nodes. In this LBDD straight forward mechanism is followed.

The drawbacks in this method are,

It suffers from flooding problem which cause significant increase in overall energy consumption. In which flooding is the way to distribute routing information updates quickly to every node in a large network.

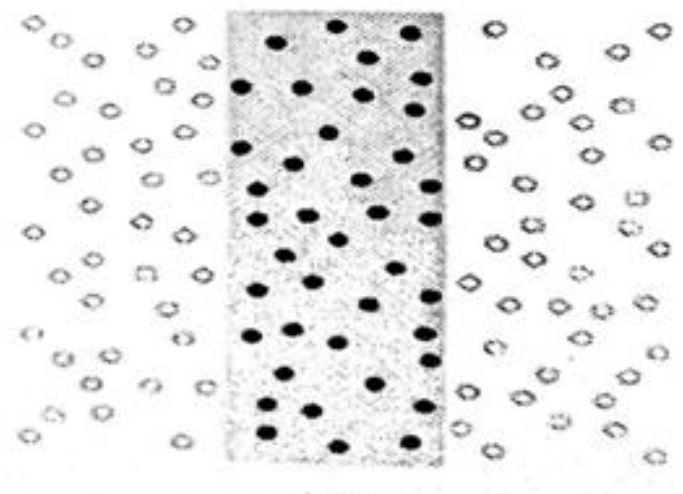

\section{(d)}

Figure 2.1: Line Approach

\subsection{Quadtree (QDD) Approach}

QDD stands for Quad Tree based data dissemination protocol, by partitions of networks in to successive quadrants.

The quadrants are further divided in to smaller quadrants. The overhead that is present in the quad tree approach is minimum as compared to the other approaches.

The drawbacks present in this approach are,

This method suffers from the hotspot problem, sensor nodes near to the base station are critical for the lifetime of sensor network because these nodes need to transmit more data than nodes away from base station. The structure of this approach is not flexible. 


\section{International Journal of Science and Research (IJSR) ISSN (Online): 2319-7064}

Index Copernicus Value (2013): 6.14 | Impact Factor (2015): 6.391

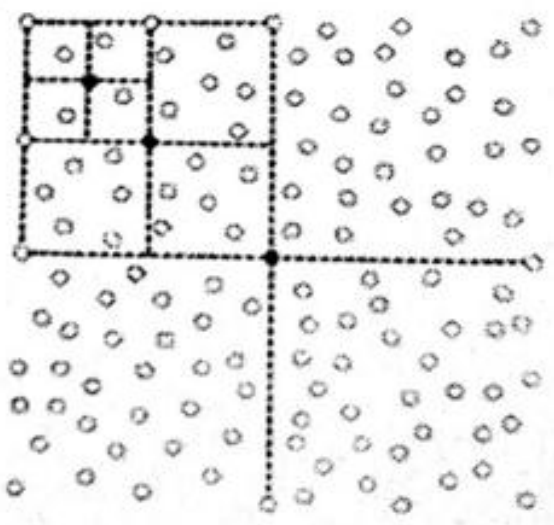

(c)

Fig 2.2 quadtree approach

\subsection{Rail Road Approach}

Rail road constructs a structure called the rail, in which the strip of nodes are in closed loop.The nodes on the rail are called rail nodes. The sensor node data sends information to the node which is the nearest rail node. When the sink node is ready to collect data from the sensor nodes, that is when it comes nearer to the railroad all the sensor nodes will become active and results in wastage of energy.

The drawback in this approach it suffers from protocol overhead, resulting in delay and excess use of resources.

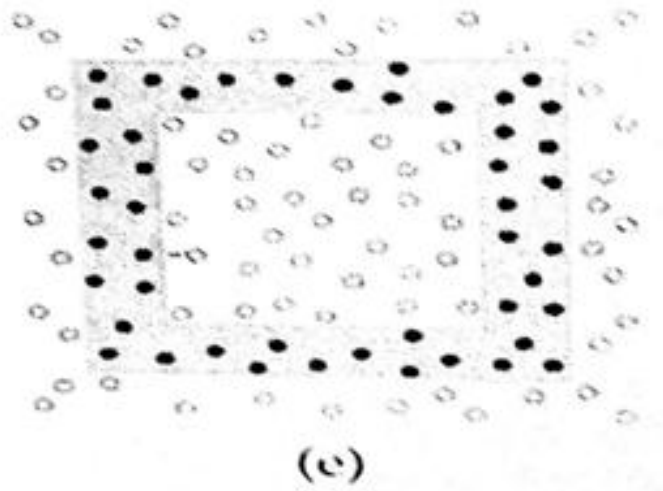

Figure 2.3: Rail Road Approach

\section{Proposed Method}

Proposed system introduced a Ring Routing mechanism, a hierarchical routing protocol for wireless sensor networks with a mobile sink. The protocol imposes three roles on sensor nodes:(i) Ring nodes (ii) The Regular nodes (iii)anchor nodes. The three sensor roles are not fixed on its roles, meaning that sensor nodes can change their functions while operating in the wireless sensor network. The location of the sink node is found through a ring node and is shared between all the ring nodes. Then the source node with the data forwards it to the sink.

\subsection{Ring Routing with Single Mobile Sink}

Ring routing it establishes a ring structure. The ring is formed with a certain radius from the centre node .It can able to form the ring structure with the node which has higher energy, ring can be easily changed. The ring consists of a node-width distance of one, strip of nodes that is closed are called the ring nodes. The shape of the ring may not be perfect as long as it forms a closed loop. After the deployment of the WSN, the ring is initially constructed by the following mechanism: An initial radius for the ring is determined.

The nodes nearer to the ring, that is defined by this radius and the center of network, by a certain threshold point are determined to be ring node candidates. From the starting of certain node (e.g. the node closest to the leftmost point on the ring) by geographic forwarding in a certain direction (clockwise/counter clockwise), the ring nodes are selected in a greedy manner until the starting node is reached and is complete with the closed loop. If the starting node is not at a reachable distance, the procedure is repeated with selecting different neighbours at every hop. If after a certain number of trials the ring cannot be created, then the radius is set to a different value and the procedure above is repeated. The advantages of this approach are given

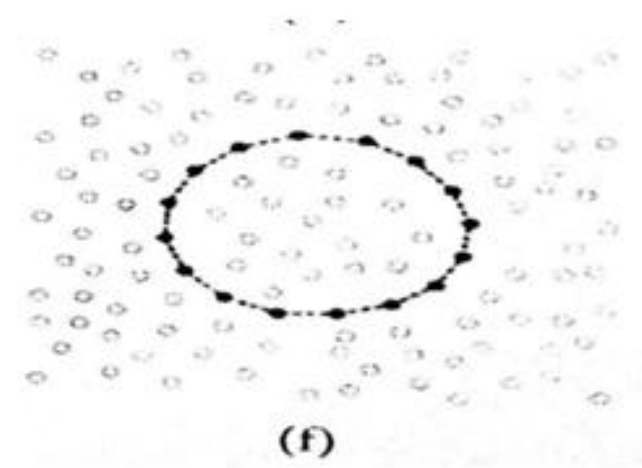

Figure 3.1: ring routing approach

- Protocol overhead is very low.

- Ring structure is flexible.

- Sink mobility pattern is random.

\subsection{Procedure for Creating Sink Position Advertisement}

When the sink moves, it selects anchor nodes (ANs) with in its neighbors. The AN serves as a delegate managing the communications between the sink and the sensor nodes. As a first step, the sink chooses the closest node (e.g., the node with the greatest SNR value) as its anchor, and broadcast an Anchor node Selection ANS packet. Before the sink leaves the communication range of the AN, it selects a new anchor nodes and informs about the position of old anchor node and the MAC address of the new AN by another ANS packet. Since now the old Anchor node knows about the new Anchor node packet, it can relay any data which is designed for it to the new AN. The current Anchor relays data packets directly to the sink node. This mechanism is referred to as the follow-up mechanism. The Anchor selection and follow up mechanism are based on the progressive footprint chaining [7]. Progressive AN selection provides a challenge in terms of find out when and how a new AN should be selected, which is closely dependent on continuous link quality estimation. Although in ideal radio channel conditions, distance to the neighboring nodes, calculated via their geographic coordinates, may be suggestive of the status of the link, it is rarely the case, not only the distance factor 


\section{International Journal of Science and Research (IJSR) \\ ISSN (Online): 2319-7064 \\ Index Copernicus Value (2013): 6.14 | Impact Factor (2015): 6.391}

affects the radio link quality it can be affected by many factors. One of the more resilient methods of link quality estimation is beaconing. In this approach, the sink broadcasts periodically beacon messages, and a link quality estimation metric (e.g., RSSI) is calculated from the reply messages originating from the neighboring nodes. Depending on the value of this metric, the sink node takes decision whether to change the current AN and which node to select as the new AN. The period of the beacon messages should be tune according to the mobility and speed of the sink, which are assumed to be known since the sink by itself takes the mobility decisions.

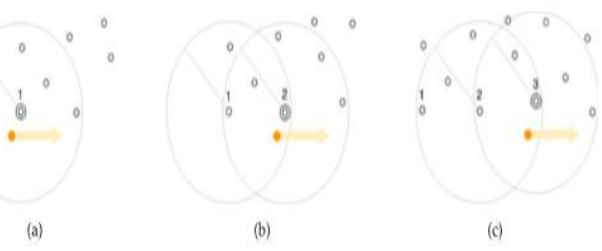

Anchor node (AN) selection: (a) time instance 1, AN: Node 1, (b) time instance 2, new AN: Node 2, and (c) time instance 3, new AN: Node 3. Figure 3.2: advertisement of sink position

\section{Architecture Diagram}

Starting from a certain node (e.g. the node nearby to the leftmost point on the ring) by geographic forwarding in a certain direction (clockwise/counter clockwise), the ring nodes are selected in a greedy manner until the starting node is reached and the loop is complete closed loop. If the starting node cannot be reached, the procedure is repeated with selection of different neighbors at each hop. If after a certain number of trials the ring cannot be formed, the radius is set to many values and the procedure above is repeated.

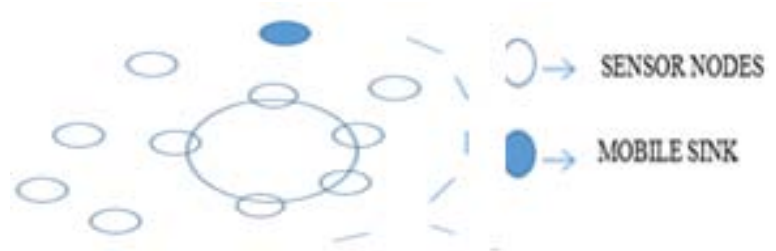

Figure 4.1: ring architecture

\section{Software Specification}

\subsection{About NS-2}

NS-2 is an open source simulation tool running on operating system like Unix. It is a discrete event simulator targeted at network research and provides considerable support for simulation of routing, multicast protocols and IP protocols, such as UDP, RTP and SRM,TCP over wired, wireless and satellite networks. It has many advantages that make it a useful tool, such as support for several protocols and the capability of graphically detailing network traffic. Additionally, NS-2 supports numerous algorithms in queuing and routing. LAN routing and broadcasts are part of routing algorithms. Queuing algorithm includes fair queuing algorithm and deficit round robin then first in first out algorithm.

NS-2 started as a variant of the REAL network simulator. Network simulator such as REAL which originally intended for studying the dynamic behavior of congestion control schemes and flow schemes in packet-switched networks. In 1995 ns development was supported by Defense Advanced Research Projects Agency (DARPA) through the VINT project at Xerox PARC, UCB,LBL and USC/ISI.

\subsection{Performance Evaluation}

The comparison is done for various hierarchical approaches. Life time comparison is done for various nodes i.e. Totally 30 nodes for ring routing, LBDD, and rail road. Ring Routing slightly in most cases while Railroad has the worst performance for all the different approaches. This behavior is due to the ANPI request/response mechanism employed by Railroad and ring routing approach. LBDD send data packets directly to the line which relays them to the sink, thus eliminating the response waiting time of delay cost to an ANPI request.

The delay comparisons for various nodes $(n=30)$, is done for various approaches such as ring routing, rail road and LBDD approaches.

The total delay for data deliveries are broken down into two different components. The ANPI request/response delay per data component is the time until a response to the ANPI request is received by the source node. The second component is the actual data dissemination delay of the path from the source to the sink.

The major two components of Ring Routing's data delivery delays are compared with LBDD's average reporting delay. The delay cost of the request or response mechanism is apparent. The actual data dissemination delay of Ring Routing is very lower than LBDD approach reporting delays.

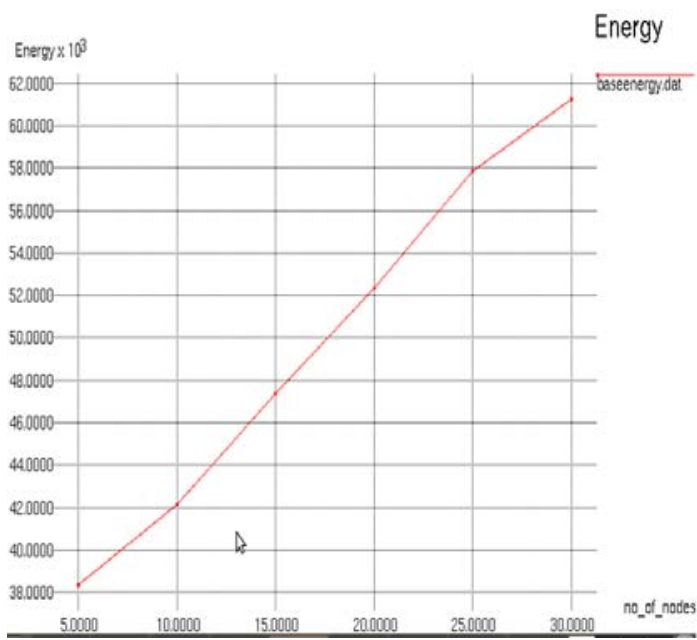

Figure 5.1: Energy comparison for various number of nodes 


\section{International Journal of Science and Research (IJSR)}

ISSN (Online): 2319-7064

Index Copernicus Value (2013): 6.14 | Impact Factor (2015): 6.391

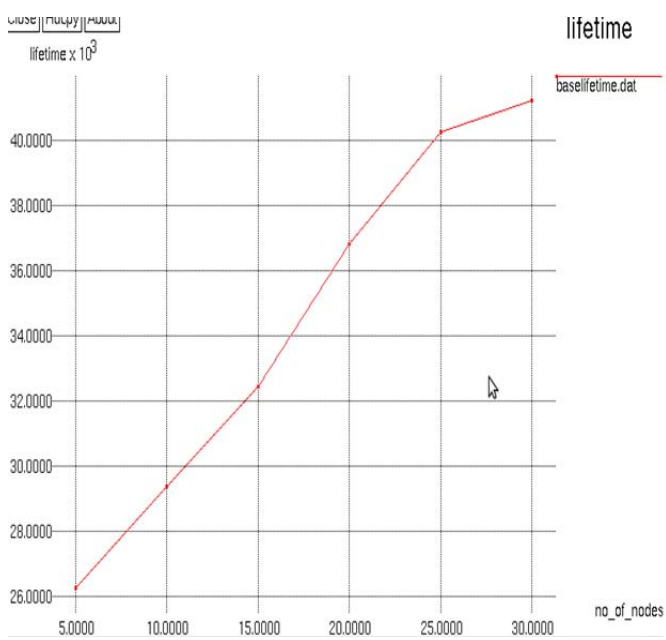

Figure 5.2: lifetime comparison for various number of nodes

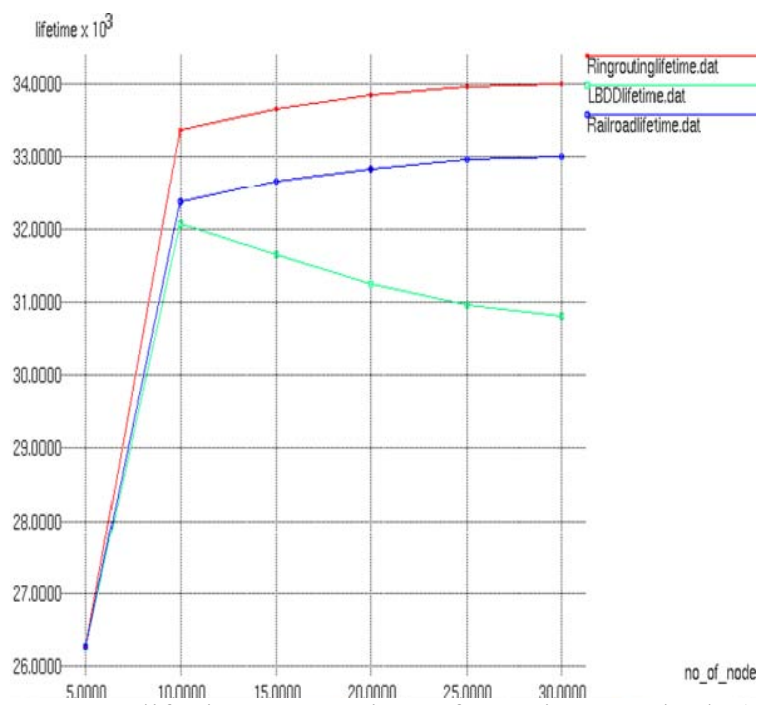

Figure 5.3: life time comparisons for various methods $(n=$ 30) node

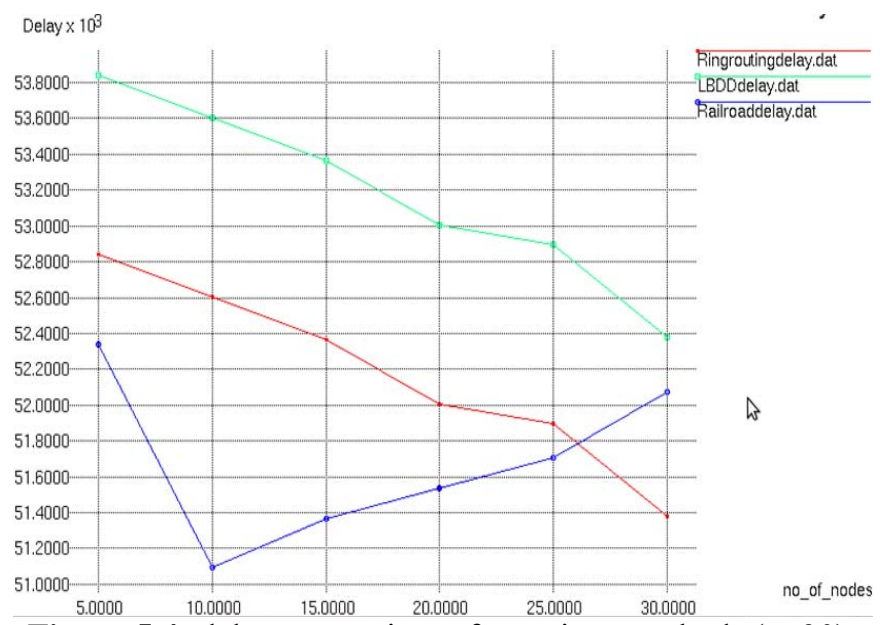

Figure 5.4: delay comparisons for various methods $(n=30)$ nodes

\section{Conclusion}

A novel mobile sink routing protocol is proposed Ring Routing, by both considering the benefits and the drawbacks of the protocols which exists in the literature. Ring Routing is a hierarchical routing protocol based on a virtual ring structure which is designed to be easily accessible and easily reconfigurable. The design requirement of our protocol is to mitigate the hotspot problem (nodes nearer to the transmitter node) observed in the hierarchical routing approaches and minimize the data reporting delays taking into consideration of various mobility parameters of the mobile sink. The performance of Ring Routing is evaluated extensively by simulations conducted in the network simulation environment. A wide range of different scenario with varying network sizes and sinks speed values are defined and used. Comparative performance evaluation results of Ring Routing with two different efficient mobile sink protocols Railroad and line based data dissemination which are also implemented in NS 2, are provided. The results show that Ring Routing definitely is an energy efficient protocol that extend the network lifetime. The reporting delays are confined within reasonable limits which proves that Ring Routing is appropriate for time sensitive applications.

In the future, we can modify Ring Routing to support multiple mobile sinks, and clustering approach is used along with it for large wireless sensor network to mitigate traffic and congestion problem.

\section{References}

[1] M. Buettner, G. V. Yee, E. Anderson, and R. Han, "XMAC: A short preamble mac protocol for duty-cycled wireless sensor networks," in Proc. 4th Inter. Conf. Embedded Network Sensor Syst., ser. SenSys '06. New York, NY, USA: ACM, 2006, pp. 307-320.

[2] I. Chatzigiannakis, A. Kinalis, and S. Nikoletseas, "Efficient data propagation strategies in wireless sensor networks using a single mobile sink," Comput. Commun., vol. 31, no. 5, pp. 896-914, 2008.

[3] Can Tunca, SinanIsik, Mehmet YunusDonmez, and CemErsoy,"Ring Routing: An Energy-Efficient Routing Protocol for Wireless Sensor Networks with a Mobile Sink, 'IEEE transactions on mobile computing, vol. 14, no. 9 , september 2015.

[4] M. Di Francesco, S. K. Das, and G. Anastasi, "Data collection in wireless sensor networks with mobile elements: A survey," ACM Trans. Sens. Netw., vol. 8, no. 1, pp. 1-31, 2011.

[5] D. K. Goldenberg, J. Lin, A. S. Morse, B. E. Rosen, and Y. R. Yang, "Towards mobility as a network control primitive," in Proc. $5^{\text {th }}$ ACM Int. Symp. Mobile ad hoc Networ. comp., ser. MobiHoc '04. New York, NY, USA: ACM, 2004, pp. 163-174.

[6] A. Gopakumar and L. Jacob, "Localization in wireless sensor net- works using particle swarm optimization," in Proc. IET Int. Conf. Wireless, Mobile Multimedia Netw., 2008, pp. 227-230.

[7] R. Jaichandran, A. Irudhayaraj, and J. Raja, "Effective strategies and optimal solutions for hot spot problem in wireless sensor networks (WSN)," in Proc. 10th Int. Conf. Inf. Sci. Signal Process. Appl., 2010, pp. 389 392.

[8] I. Kang and R. Poovendran, "Maximizing static network lifetime of wireless broadcast ad hoc networks," in 
Proc. IEEE int. Conf. Commun., vol. 3, 2003, pp. 22562261.

[9] W. Liang, J. Luo, and X. Xu, "Prolonging network lifetime via a controlled mobile sink in wireless sensor networks," in Proc. IEEE Global Telecommun. Conf., 2010, pp. 1-6.

[10]K. Lin, M. Chen, S. Zeadally, and J. J. Rodrigues, "Balancing energy consumption with mobile agents in wireless sensor networks," Future Generation Comput. Syst., vol. 28, no. 2, pp. 446- 456, 2012.

[11] C.-J. Lin, P.-L.Chou, and C.-F. Chou, "HCDD: Hierarchical cluster-based data dissemination in wireless sensor networks with mobile sink," in Proc. Int. Conf. Wireless Commun. Mobile Comput., 2006, pp. 1189-1194.

[12]X. Li, J. Yang, A. Nayak, and I. Stojmenovic, "Localized geographic routing to a mobile sink with guaranteed delivery in sensor networks," IEEE J. Sel. Areas Commun.., vol. 30, no. 9, pp. 1719-1729, Sep. 2012.

[13] J. Luo and J.-P.Hubaux, "Joint mobility and routing for lifetime elongation in wireless sensor networks," in Proc. INFOCOM 24thAnnu.Joint Conf. IEEE Comput.Commun. Soc., vol. 3, 2005, pp. 1735-1746.

[14]D. Moss, and P. Levis, "BoX-MACs: Exploiting physical and link layer boundaries in low-power networking," Stanford Univ., Stan- ford, CA, USA, Tech. Rep. SING-08-00, 2008.

[15] D. Niculescu, "Positioning in ad hoc sensor networks," IEEE Netw., vol. 18, no. 4, pp. 24-29, Jul. 2004.

[16] S. Oh, Y. Yim, J. Lee, H. Park, and S.-H. Kim, "Nongeographical shortest path data dissemination for mobile sinks in wireless sen- sor networks," in Proc. IEEE Veh. Technol. Conf., Sep. 2011, pp. 1-5.

[17] S. Olariu and I. Stojmenovi, "Design guidelines for maximizing lifetime and avoiding energy holes in sensor networks with uniform distribution and uniform reporting," in Proc. IEEE INFOCOM, 2006, pp. 1-12.

\section{Author Profile}

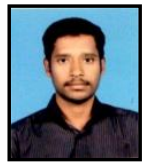

Sudharson. S received his M.E degree in communication systems from Sri Krishna college of engineering and technology, affiliated to Anna university, Coimbatore. His research interests include wireless sensor networks, network protocol design.

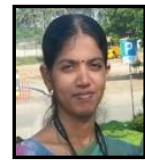

Jennie Bharathi.R is currently working as a assistant professor in Sri Krishna college of engineering and technology, affiliated to Anna university, Coimbatore. networks Her research interests include internet protocol, sensor 